\title{
Dinámicas locales en torno al cultivo de hoja de coca: elementos para el estudio desde el mercado ilegal de la cocaína ${ }^{+}$
}

\author{
NICOLÁS ZEVALLOS TRIGOSO* \\ Pontificia Universidad Católica del Perú \\ nzevallos@pucp.edu.pe \\ https://doi.org/10.18800/rcpg.201701.001
}

\section{RESUMEN}

La autonomía del Estado para alcanzar los objetivos de las políticas se ve afectada por su relación con los actores sociales. La dinámica del mercado de la cocaína ofrece una arena importante para entender cómo estas relaciones afectan el control de los mercados ilegales. Los actores involucrados en la cadena de suministro de este mercado ilícito, despliegan sus recursos para limitar la autonomía del Estado y afectar la efectividad de la política de drogas. Con base en una revisión de estudios recientes, es posible proponer algunas ideas para comprender la configuración de estas relaciones en el marco del control del cultivo de coca en el Perú y la región andina. Esto significa entender que (1) la dinámica de esta actividad agrícola $-\mathrm{y}$ su valoración como problema público — está fuertemente asociada con la racionalidad económica del mercado de la cocaína; (2) que esta racionalidad tiene gran importancia en la defensa histórica del cultivo de coca; y (3) que, hoy en día, las tensiones entre el Estado y los actores sociales involucrados en el cultivo de coca en Perú se basan principalmente en estrategias de control y defensa a nivel local.

Palabras clave: mercados ilegales, política de drogas, capacidades estatales, cocaína, hoja de coca.

\footnotetext{
* Investigador principal del Laboratorio de Criminología, grupo de investigación adscrito a la Escuela de Gobierno y Políticas Públicas de la Pontificia Universidad Católica del Perú.

** El presente artículo introduce el volumen de la Revista de Ciencia Política y Gobierno dedicado a las capacidades estatales y la implementación de políticas públicas. Específicamente, aborda el impacto de las relaciones entre el Estado y los actores sociales y políticos, en el control de la producción del cultivo de hoja de coca en el Perú. En esta revisión se integran las presentaciones de los artículos que componen el volumen temático.
} 
Local dynamics in the sideline of coca leaf cultivation: study elements of the illegal cocaine market

\section{Abstract}

The autonomy of the State to achieve policy goals is affected by its relation with the social actors. The dynamics of the cocaine market offers an important arena to understand how this relations affect the control of illegal markets. The actors involved in the supply chain of this illicit market, deploy its resources in order to limit the autonomy of the State, and to affect the effectiveness of the drug policy. Based on a review of recent studies, it is possible to propose some ideas in order to understand the configuration of this relations in the sideline of the control of the coca cultivation in Perú and the Andean Region. This means understanding that (1) the dynamic of this agricultural activity — and its valuation as a public problem-is strongly associated to the economic rationality of the cocaine market; (2) that this rationality has great importance in the historical defense of the coca cultivation; and (3) that, nowdays, the tensions between the State and the social actors involved in the cultivation of coca in Perú, are maily based on control and defense strategies at a local level.

Keywords: illicit markets, drug policy, state capacity, cocaine, coca leaf. 


\section{Presentación}

El presente artículo se inserta en el debate sobre las capacidades estatales y la implementación de políticas públicas. Específicamente, aborda el impacto de las relaciones entre el Estado y los actores sociales y políticos, en las condiciones de posibilidad para el logro de los objetivos de una política pública. Esto parte de la mirada de la dimensión relacional de las capacidades estatales y que refiere a la autonomía del Estado respecto a otros actores políticos (Giraudy, 2012, p. 601). Así, se entiende que las relaciones entre el Estado y los actores sociales y políticos involucrados en un problema público condicionan la autonomía del Estado, lo cual influye en la posibilidad de que este logre los objetivos de política que se propone.

Para abordar este tema se plantea estudiar el control del mercado ilegal de la cocaína en el Perú, aterrizando en la exploración de las medidas para el control de los cultivos de hoja de coca. Como materia prima elemental para la producción de derivados cocaínicos, la hoja de coca se encuentra controlada por el Estado peruano. Para ello, dispone de medidas con las que se procura la regulación de la producción lícita de esta planta, la erradicación de los cultivos ilícitos, así como la implementación de proyectos de desarrollo económico y productivo en las zonas donde esta actividad tiene presencia.

Las medidas de control de cultivos de hoja de coca no son ajenas a tensiones entre el Estado y los actores involucrados en esta actividad agrícola. Estas pueden responder a reivindicaciones culturales, pero también a la preocupación por los aspectos materiales a los que acceden gracias a la misma. En algunos casos, las resistencias derivadas de estas tensiones han impedido la implementación de estas medidas. En línea con el debate sobre la dimensión relacional de las capacidades estatales, ello pone en discusión las condiciones de posibilidad y la autonomía para que el Estado logre el pretendido control del mercado ilegal de la cocaína.

En ese horizonte, el objetivo del presente artículo es plantear algunas consideraciones sobre los sentidos que subyacen a las relaciones entre el Estado y los actores involucrados en el cultivo de hoja de coca, derivadas de la implementación de medidas de control de esta actividad. Para tal fin, se propone describir la asociación de la hoja de coca con el mercado ilegal de la cocaína, caracterizar las relaciones entre el Estado y los actores involucrados en este cultivo, así como proponer algunas ideas para la discusión sobre estas relaciones entre el Estado y los actores a nivel local.

Las consideraciones planteadas parten de tres ideas complementarias. En primer lugar, que el cultivo de hoja de coca se encuentra estrechamente 
asociado a la demanda del sistema productivo del mercado ilegal de la cocaína. En segundo lugar, que la defensa del cultivo de hoja de coca responde, en gran medida, a su aprovechamiento material, a pesar de su articulación al citado sistema productivo ilegal. En tercer lugar, que actualmente es posible observar (1) una concentración de estas tensiones a nivel local en las que (2) el Estado aparece como un actor más con necesidad de expandirse y dialogar con los actores locales para lograr sus objetivos de política.

El presente documento está dividido en cuatro secciones. La primera trata sobre la asociación del cultivo de la hoja de coca con el mercado ilegal de la cocaína, y plantea entender de qué manera este último impulsa la dinámica de la citada actividad agrícola. La segunda sección discute la compartimentalización y descentralización de las relaciones entre el Estado y los actores involucrados en la cadena de suministro de cocaína, y aterriza en los sentidos que han dado forma a la defensa del cultivo en la región andina. A partir de una revisión de literatura sobre la materia, la tercera sección propone algunas ideas para comprender cómo funcionan las relaciones entre el Estado y los actores locales en la defensa del cultivo de hoja de coca en el Perú. A manera de cierre, la cuarta sección plantea una serie de consideraciones para el estudio del mercado ilegal de la cocaína, de la defensa del cultivo de hoja de coca, así como de la política de drogas.

\section{El CULTIVO DE HOJA DE COCA Y EL MERCADO ILEGAL DE LA COCAÍNA}

Si se observan los contextos de uso, las valoraciones y el control de la hoja de coca (y sus derivados) a lo largo de la historia, es posible identificar cuando menos cuatro estadios. El primero refiere al periodo prehispánico, en el que la hoja de coca como planta sagrada era reservada para el consumo ritual y político de las élites (de la Peña, 1972; Hinostroza, 1985; Morales, 2002). El segundo implica el tránsito hacia la Colonia, en la que fue utilizada para garantizar el trabajo de los indios en las minas de plata en condiciones extremas (Lloyd y Lloyd, 1913; Cabieses 1996; García 2008). El tercero refiere a la Industrialización, en el que se incorpora la cocaína, ampliamente difundida por la industria farmacéutica, como maravilla médica (Cabieses, 2005; Gootenberg, 2003, 2006). El cuarto y último estadio implica la fiscalización de este bien de consumo global, al ser asociado con riesgos tanto para la salud, la seguridad como la gobernabilidad democrática (Gootenberg, 2003; ONU, 1961, 1988).

Un hilo conductor en esta historia de la hoja de coca y de los derivados producidos a partir de la misma, es el tránsito desde su uso ritual y religioso 
hacia la explosiva expansión global de su aprovechamiento material como bien de consumo. Es en este tránsito que progresivamente se construye la valoración problemática de este producto vegetal y sus derivados. Desde la Colonia, el uso instrumental de la hoja de coca fue entendido por la Iglesia católica como un problema para la evangelización de los indios (Cabieses, 1996, p. 150). Durante la Industrialización, la comunidad científica planteó reservas sobre los efectos nocivos del uso desmedido de cocaína, promocionado por la industria del ocio (Gootenberg, 2003, p. 9). Constituido como un bien de consumo global proscrito, el manejo por redes criminales de un mercado de la cocaína altamente rentable, sustenta las políticas de control en los países a lo largo del planeta (Garzón, 1993, p. 202).

A partir de estas premisas, resulta importante entender que esta valoración problemática de la hoja de coca y sus derivados responde a su progresiva configuración como bienes de consumo global. Ello permite sostener que la racionalidad económica de este fenómeno trasciende a su carácter ilegal, puesto que este carácter deriva de una reacción ante los problemas que conlleva su expansión como actividad económica. Con ello, es posible incorporar a la discusión ideas como la ya mencionada de «mercado ilegal». La literatura entiende que tales mercados constituyen arenas de intercambio de bienes y servicios por dinero, en las que los bienes y los servicios por sí mismos, o la producción y comercio de estos, se encuentra proscrito por la ley penal (von Lampe, 2016, p. 81). Asimismo, también es posible sumar al análisis conceptos como el de "cadena de suministro", entendiendo por tal a los sistemas integrados por personas y organizaciones responsables de transformar la materia prima en un bien y de su distribución hacia los consumidores (LINCS, 2016, p. 1).

Estas ideas ofrecen un marco para estudiar la articulación del cultivo de hoja de coca a la cadena de suministro del mercado ilegal de la cocaína. La literatura reconoce que esta actividad agrícola es el punto de partida de una secuencia de actividades productivas y comerciales que permiten la transformación de esta planta en un estupefaciente (Allen, 2005; Kennedy, Reuter y Riley, 1993). El concepto de cadena de suministro permite entender que este circuito está compuesto por eslabones que integran actividades de abastecimiento de materias primas, de producción de derivados, así como de comercio mayorista y al menudeo de estos derivados, como se sugiere en el modelo expuesto en el flujograma 1. Desde esta perspectiva, el abastecimiento de hoja de coca implica un conglomerado de tareas y operaciones específicas para esta actividad agrícola: acceso a campos de cultivos, semillas y plantas, agroquímicos, mano de obra agrícola, etcétera. Asimismo, integra a una serie de actores cuyos inte- 
reses (especialmente económicos) dinamizan la articulación de este producto vegetal con el mercado ilegal de la cocaína.

Flujograma 1. Modelo de la cadena de suministro del mercado ilegal de la cocaína

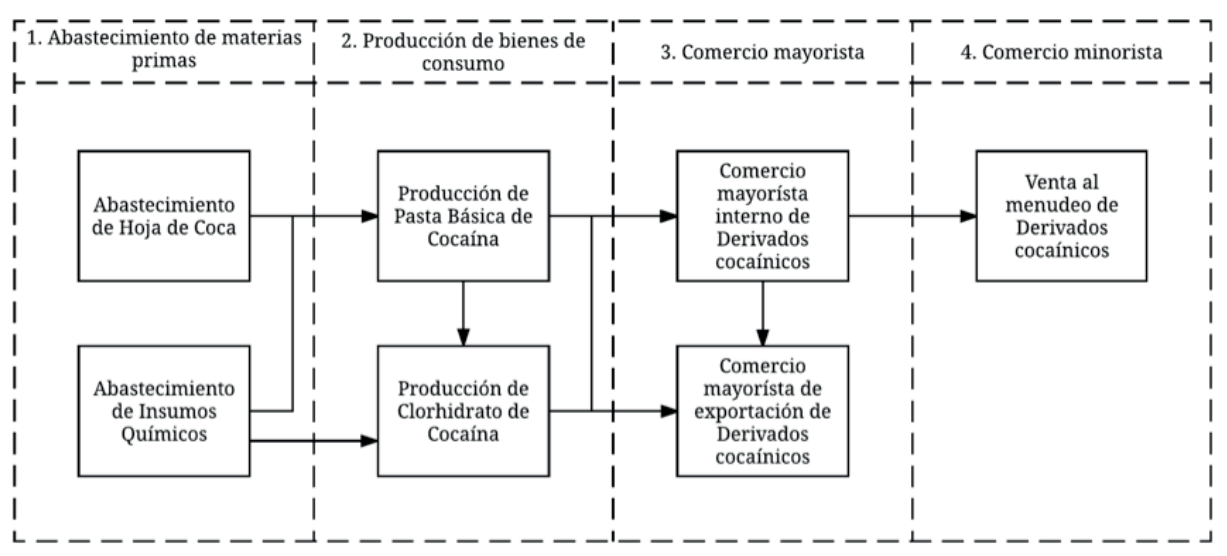

Algunas evidencias dan cuenta de la importancia de la cadena de suministro del mercado ilegal de la cocaína para la dinámica del cultivo de hoja de coca. Para el año 2013, la producción potencial estimada de hojas de coca en el Perú alcanzó las 121242 toneladas métricas (UNODC, 2016, p. 7). Se estima que la demanda de hoja de coca para el consumo tradicional, en ese mismo año, llegó a las 10728 toneladas métricas (INEI, 2015, p. 15). Una operación aritmética simple permite sugerir que las 110514 toneladas métricas de hoja de coca restantes (el 91,2\% del total) se destinaron a abastecer la cadena de suministro del mercado ilegal de la cocaína. Simulando la venta de todo este volumen al precio promedio ponderado en parcela de la hoja de coca secada al sol (US\$ 3,6 por kilogramo a 2013, según UNODC, 2014, p. 7), esta transacción sumaría cerca de US\$398 millones.

Es importante contrastar este último monto con el movilizado en las otras actividades de la cadena de suministro. Para tal fin resulta útil un ejercicio de simulación de la venta del total de cocaína que se podría producir con el ya señalado volumen de hoja de coca que se destinaría ilícitamente para este fin. Según el último factor de conversión conocido, este volumen de cocaína alcanzaría las $294 \mathrm{tm}^{1}$. Como se observa en el gráfico 2, si bien la venta de la hoja

Al respecto, ver López (2015, p. 64). 


\section{Gráfico 1. Destino de la producción de hoja de coca en el Perú a 2013 (en tm)}

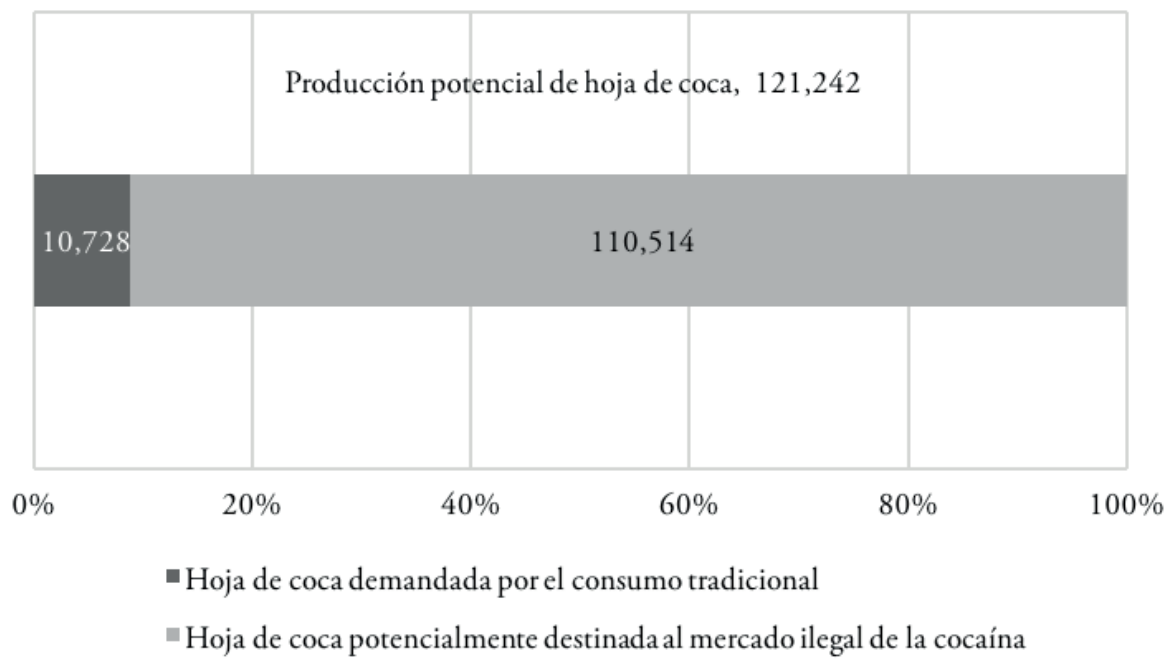

Fuente: Elaboración propia a partir de UNODC (2014) e INEI (2015).

de coca sería 3\% mayor que la de la cocaína en zonas de producción, significaría el 90\% de esta venta en zonas de exportación (el puerto del Callao, por ejemplo). Asimismo, representaría el 3\% de la venta al por mayor en España, y el 1,78\% de la venta al menudeo en el mismo país. Sobre esto último, cabe señalar que la pureza de la cocaína en Espańa alcanza el 63\% en la venta mayorista y el $41 \%$ en la venta al menudeo, lo que sugiere que el monto logrado con estas ventas es superior al estimado en este ejercicio.

Como se ha señalado, la cadena de suministro del mercado ilegal de la cocaína es importante en la dinámica del cultivo de hoja de coca. Sin embargo, el ejercicio anterior sugiere que el abastecimiento de hoja de coca no es la actividad que más dinero moviliza en la citada cadena de suministro, siendo bastante menor frente a las actividades comerciales. La literatura plantea que el incentivo para esta dinámica se encuentra en las facilidades para el acceso a un mercado relativamente estable, antes que en la rentabilidad: pagos por adelantado, compra directa en el campo de cultivo, precios competitivos (Zevallos, 2016b). Sobre este último punto, como se observa en el gráfico 3, entre enero del año 2000 y diciembre de 2016, el precio promedio nacional de la hoja de coca ha mostrado una tendencia incremental, oscilando entre los US\$1,5 y los US\$ 4,4 por kilogramo. 


\section{Gráfico 2. Estimación del monto de la venta simulada de la cocaína potencialmente producida con la producción de hoja de coca en el Perú a 2013 (en millones US\$)}

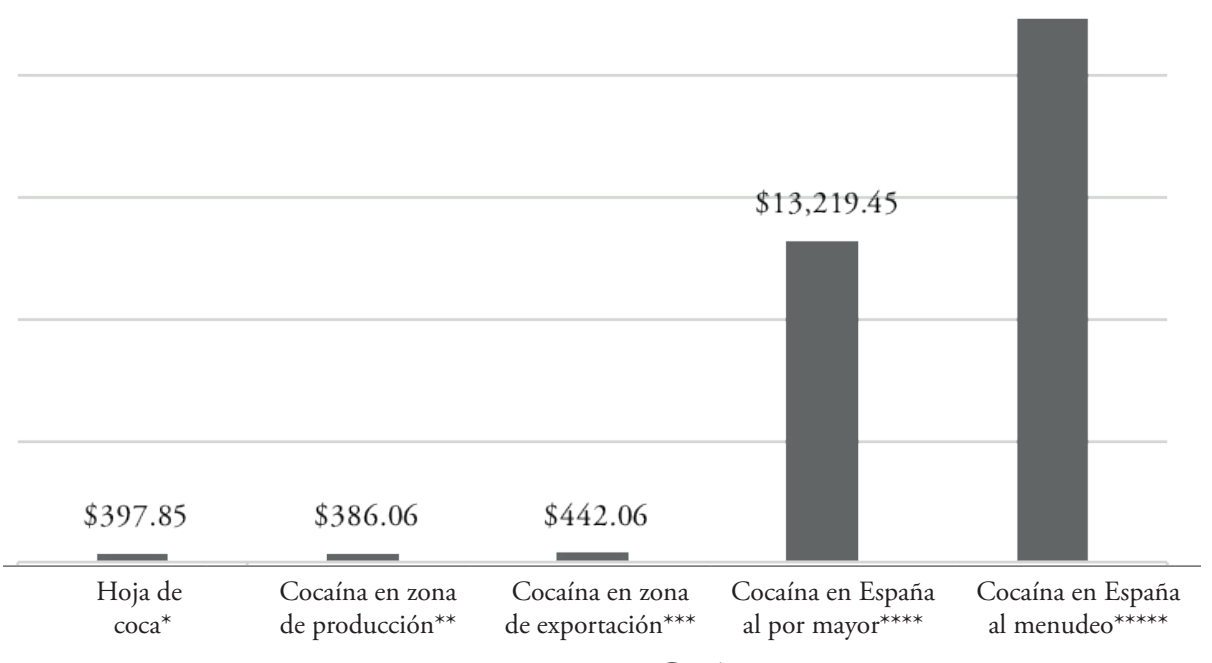

Cocaína

Elaboración propia a partir de UNODC (2014); López (2015); Mella (2012); Estadísticas UNODC.

*Estimado de la venta del volumen de hoja de coca potencialmente producido, al precio promedio ponderado en parcela a 2013 (US\$3,6/kg según UNODC, 2014).

**Estimado de la venta del volumen potencialmente producido con la hoja de coca producida a 2013, al precio promedio de clorhidrato de cocaína en zonas de producción (US\$1310/kg según UNODC, 2014).

***Estimado de la venta del volumen potencialmente producido con la hoja de coca producida a 2013, al precio promedio de clorhidrato de cocaína en zonas de exportación (US\$1500/kg según Mella, 2012).

****Estimado de la venta del volumen potencialmente producido con la hoja de coca producida a 2013, al precio promedio de clorhidrato de cocaína a 2014 al por mayor (US\$44857/kg, según Estadísticas de UNODC, consultadas al 28 de mayo de 2017).

*****Estimado de la venta del volumen potencialmente producido con la hoja de coca producida a 2013, al precio promedio de clorhidrato de cocaína a 2014 al menudeo (US\$ 75/g, según Estadísticas de UNODC, consultadas al 28 de mayo de 2017).

A nivel global, el mercado de la cocaína también ha mostrado relativa estabilidad. A pesar de las variaciones, el volumen disponible para el consumo de cocaína para el año 2014 (537 tm) es semejante al del año 1998 (512 tm). Si bien en este periodo se registra una reducción en el promedio de consumo anual per cápita de cocaína, la estabilidad del volumen disponible parece responder a un incremento en el número anual de consumidores, que pasa de 14 millones a 18.8 millones (UNODC, 2016, p. 41). Una explicación para ello es el incremento del consumo de derivados cocaínicos en Brasil — particularmente el crack elaborado con pasta básica de cocaína - durante los últimos años 
Gráfico 3. Evolución del precio promedio mensual de la hoja de coca, enero 2000 - diciembre 2016

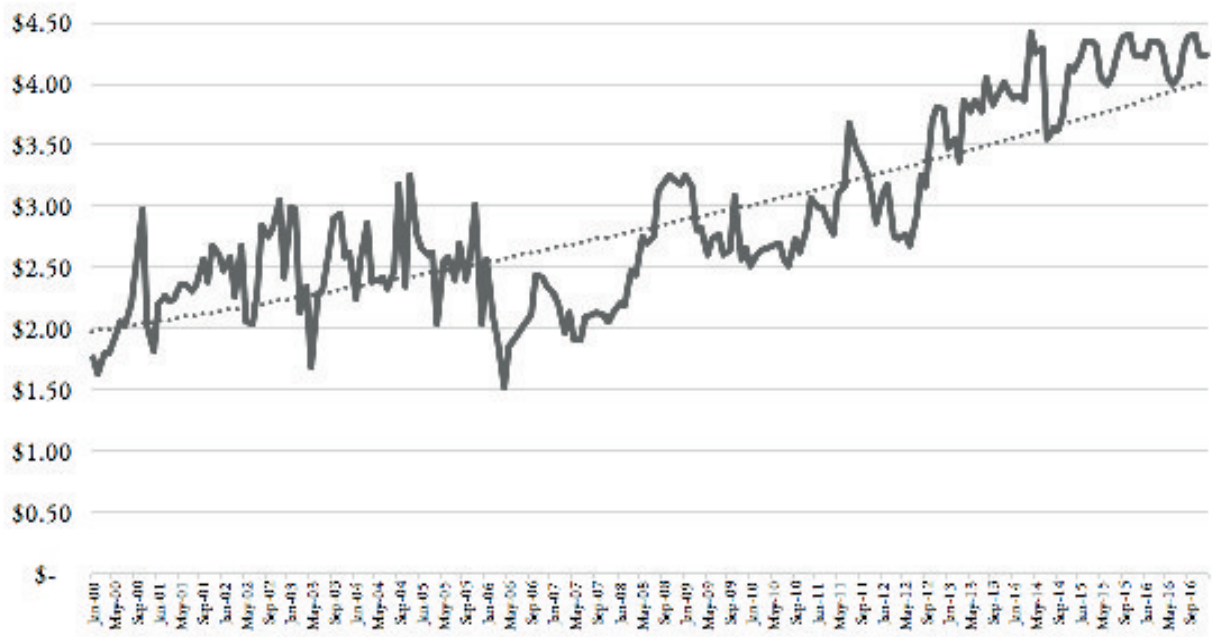

Elaboración propia con información proporcionada por el Observatorio Peruano de Drogas al 28 de mayo de 2017.

(UNODC, 2016). Así, este país, separado de los países productores por una extensa frontera amazónica muy permeable y difícil de controlar, emerge como un nuevo polo atractor más cercano para la cadena de suministro del mercado ilegal de la cocaína.

En síntesis, las evidencias sugieren que la problematización de la hoja de coca y sus derivados está asociada a la histórica expansión de su aprovechamiento material. Asimismo, que la cocaína se constituye como un importante bien de consumo cuyo mercado ilegal tiene alcance global, a pesar de las prohibiciones. Por otro lado, que los incentivos para el abastecimiento de hojas de coca para la cadena de suministro del mercado ilegal de la cocaína no necesariamente derivan de la rentabilidad, sino de la accesibilidad a un mercado relativamente estable. Este horizonte sugiere comprender las relaciones existentes en torno al control del cultivo a partir de la racionalidad económica que subyace a esta actividad. Desde esta perspectiva, algunos autores sugieren entender el cultivo de hoja de coca como una actividad agroindustrial cuya articulación comercial tiene alcance planetario (Del Olmo, 1975). La sección siguiente plantea algunas consideraciones sobre cómo los aspectos materiales han influido en la configuración de la defensa del cultivo de hoja de coca en los países productores. 


\section{LA DEFENSA DEL CULTIVo DE HOJA DE COCA EN LA REgión ANDINA}

Uno de los aportes de incorporar el concepto «cadena de suministro» al análisis de mercados ilegales, es que permite comprender las particularidades de las actividades que dan forma a un sistema productivo y comercial. Lo señalado va en línea con dos características que la literatura reconoce en la configuración de las redes del mercado ilegal de la cocaína. En primer lugar, se trata de actividades compartimentalizadas que, a pesar de configurar una secuencia, tienen objetivos, recursos y procedimientos específicos. En segundo lugar, y como una característica especial de la dinámica actual de estas redes, que las actividades se descentralizan territorialmente, de manera que pueden operar en distintas partes del globo y así mantener el flujo del suministro de cocaína para el mercado ilegal (Kenney, 2007). Como se verá en lo sucesivo, ambas características son centrales para comprender las relaciones existentes a lo largo de la cadena de suministro.

La compartimentalización de las actividades que componen la cadena de suministro sugiere también la necesidad de medidas de control particulares y, por ende, de relaciones en torno a las mismas. Así, no necesariamente serán iguales las relaciones en torno al control de la hoja de coca (que implican el control de una actividad agrícola), que las que se generan respecto al comercio al por mayor (que implica la interdicción de operaciones logísticas trasnacionales) o al comercio al menudeo (que significan la persecución de redes locales). Por ello, es de esperarse que las relaciones en torno a cada actividad conlleven formas distintas de resistencia de los actores involucrados y, por ende, condiciones de posibilidad diferentes para hacer efectivo el control del Estado. No será lo mismo construir una relación con gremios cocaleros que defienden el cultivo de hoja de coca, que con redes criminales que protegen la exportación de cocaína.

La descentralización de las actividades plantea un panorama semejante, aun cuando alude a la distribución geográfica de esta cadena de suministro. Factores como la disponibilidad de materias primas o la cercanía con los consumidores, influyen en que determinados territorios ofrezcan una ventaja competitiva para el desarrollo de ciertas actividades de la cadena de suministro (Thoumi, 2003, p. 55). Esto da a entender que las relaciones también se descentralizan. Así, resulta racional esperar que las resistencias al control del cultivo se concentren en las zonas de producción, mientras que las referidas a la interdicción de las actividades de exportación se ubiquen en localidades donde se desarrollan estas operaciones logísticas (puertos, por ejemplo). Como se observa, los conceptos 
de compartimentalización y descentralización de la cadena de suministro plantean aspectos aparentemente evidentes. Sin embargo, cuestionan sentidos comunes que sugieren — con poca precisión-que mercados ilegales como el de la cocaína operan a nivel global orgánicamente y bajo un mismo sentido.

Una mirada a América Latina permite tener un panorama sobre cómo se compartimentalizan y descentralizan actualmente las relaciones a lo largo de la cadena de suministro del mercado ilegal de la cocaína. Así, en México y el triángulo norte de Centroamérica se concentra la violencia asociada al control del comercio al por mayor de cocaína (y otras drogas), destinada a los Estados Unidos, el mayor consumidor de este estupefaciente. En Brasil y otros países del Mercosur, estas relaciones se vinculan con el comercio al menudeo, controlado por redes criminales en los principales centros urbanos: São Paulo, Río de Janeiro, Buenos Aires. Por su parte, en la región andina (Bolivia, Colombia y Perú) se concentran las relaciones en torno a los primeros eslabones de la cadena: las actividades productivas de derivados cocaínicos, así como las relativas al desarrollo del cultivo de hoja de coca.

Si bien las relaciones en torno a la hoja de coca en los países productores muestran algunos matices, también dan cuenta de elementos comunes. En Bolivia, el surgimiento del movimiento cocalero aparece relacionado a procesos de colonización asociados a proyectos fallidos de reforma agraria, que derivaron en el progresivo involucramiento de los colonos en esta actividad agrícola (Pinto, 2008, p. 43). En paralelo, se construyeron organizaciones sindicales integradas por cocaleros, que asumen la interlocución con el Estado para, por ejemplo, la distribución de los beneficios derivados de los programas de sustitución de cultivos (Pinto, 2008, p. 46). Este proceso fue el inicio de la construcción de un actor nacional, desde bases estrictamente sindicales (Castillo, 2004, p. 3). Esta construcción se sostiene en factores como la importancia de la economía de la coca en Bolivia (Castillo, 2004, p. 3; Cortez, 1993, p. 126). También en las tensiones generadas por la política de drogas que, según señala la literatura, no solo es entendida como una amenaza para el sustento del campesinado cocalero, sino que implicaba la militarización de las zonas de producción (Castillo, 2004, p. 4; Cortez, 1993, p. 141). En este escenario, la creación del Movimiento para la Acción Social-MAS representa la consolidación de este liderazgo cocalero en la reivindicación de la soberanía nacional (Silva, 2010, p. 37).

Por su parte, en Colombia, la emergencia del movimiento cocalero en zonas como el Putumayo, presenta como antecedentes movimientos sociales que, entre las décadas de 1970 y 1990, demandaban al Gobierno colombiano la cobertura de necesidades básicas (Ramírez, 2011, p. 86). La colonización de las 
zonas rurales tuvo como principal dinamizador al cultivo de hoja de coca, cuyo control por el Estado colombiano dio pie a diversas reacciones. Estas se centraron en tres aspectos: el cese de las fumigaciones aéreas, la no creación de zonas de emergencia (pues implicaban abusos de las Fuerzas Armadas) y que no se restrinja el acceso a recursos como el cemento y el combustible (Ramírez, 2011, p. 112). De acuerdo a la literatura, fueron estas las principales motivaciones de las marchas de gremios cocaleros en 1996 (Uribe y Ferro, 2014, pp. 65-66). Dos factores marcaron el desarrollo de este proceso. En primer lugar, la ubicuidad de las Fuerzas Armadas Revolucionarias Colombianas - FARC en las zonas con presencia de cultivos de hoja de coca (Díaz y Sanchez, 2004, pp. 35-38). Esto ha implicado su participación en el cultivo, primero oponiéndose a los mismos, para luego tratar de regularlos por los ingresos que representaban a los campesinos, hasta llegar finalmente a proponer su sustitución en las agendas de negociación con el Estado (Uribe y Ferro, 2014, pp. 71-72). En línea con esto, en segundo lugar, la criminalización del cultivo implicó no poder incorporar al movimiento cocalero a la dinámica de los movimientos sociales campesinos, al igual que en Bolivia o Perú (Ramírez, 2011, p. 116).

El panorama en Perú comparte varios de los elementos referidos en el caso boliviano y colombiano. En primer lugar, un proceso de colonización de la franja amazónica dinamizado por el cultivo de una hoja de coca articulada al mercado ilegal de la cocaína, ante la ausencia de otras actividades económicas accesibles (CVR, 2003). En segundo lugar, la emergencia de un movimiento cocalero que reacciona ante las medidas de control con acciones de protesta, y con capacidad de generar — al menos temporalmente- una plataforma nacional de resistencia (Van Dun, 2009). Tercero, en línea con el proceso colombiano, la confluencia territorial de la actividad cocalera y el conflicto armado, protagonizado en este caso por organizaciones catalogadas como terroristas: Sendero Luminoso y el Movimiento Revolucionario Túpac Amaru - MRTA (CVR, 2003). Cuarto, una plataforma de demandas asociadas a las posibilidades económicas que representa el cultivo de hoja de coca, ante la ausencia de otras alternativas económicas viables (Zevallos, 2014).

A manera de síntesis, las ideas de compartimentalización y descentralización de la cadena de suministro del mercado ilegal de la cocaína permiten comprender las particulares relaciones que recorren este sistema productivo y comercial. En este escenario, es en la región andina en donde se concentran las relaciones relativas al control y la defensa del cultivo de una hoja de coca cuya producción, como se ha señalado ya, se destina principalmente a elaborar derivados cocaínicos. Además de las reivindicaciones culturales, los aspectos 
materiales han tenido particular protagonismo en la construcción de plataformas nacionales de defensa del cultivo en Bolivia, Colombia y Perú. Sin embargo, en el caso peruano, la latencia del movimiento cocalero, derivada de la fragmentación de los intereses, así como de la deslegitimación de las organizaciones intermediarias, ha redirigido las estrategias de defensa al espacio local. Como se verá en la sección siguiente, a este nivel el Estado se enfrenta a la necesidad de expandirse y dialogar con los actores locales, a fin de cumplir sus objetivos de política.

\section{Dinámicas locales en torno al control del Cultivo de hoja de COCA EN El Perú: elementos Para la discusión}

De acuerdo a la literatura, el nuevo panorama de las tensiones en torno al cultivo de hoja de coca en el Perú responde a la situación de latencia en la que se encuentra el movimiento cocalero, como consecuencia de (1) la fragmentación de los intereses en los gremios que las componen, así como la de (2) deslegitimación de otros operadores intermediarios, tales como los partidos políticos (Zevallos, 2016b). Inclusive el accionar de organizaciones terroristas como Sendero Luminoso, que en zonas como el Alto Huallaga se sumaban a la defensa (al menos instrumental) del cultivo, se ha visto mermado dada su desarticulación. Estos factores han implicado la poca visibilidad y capacidad de acción de lo que alguna vez constituyó una plataforma nacional de defensa de la hoja de coca. Sin embargo, los intereses en torno al cultivo se mantienen, proyectando sus resistencias desde lo local. Diversos estudios plantean elementos para comprender cómo se configuran estas dinámicas locales en torno al control del cultivo de hoja de coca en el Perú.

Para Heuser ${ }^{2}$ (en esta edición), una posible entrada para comprender cómo se configuran las tensiones en torno al control de cultivos en localidades como el valle del Monzón (ubicado en el Alto Huallaga, una de las principales cuencas cocaleras del Perú) es a partir de la idea de campos de poder. Durante décadas, el citado valle fue uno de los principales bastiones del movimiento cocalero y de la defensa política del cultivo. Eso implicó un campo de poder en el cual las reglas eran impartidas por los actores pertenecientes a este movimiento social. Sin embargo, las intervenciones estatales de los últimos años han implicado un proceso de transición de este escenario local. Esto deriva de aspectos como la

\footnotetext{
2 Los autores citados en esta edición son aquellos cuyos artículos se encuentran en este número de la Revista de Ciencia Politica y Gobierno.
} 
pérdida de espacio de Sendero Luminoso, que si bien no mantenía un vínculo orgánico con el movimiento, sí representaba un actor importante en la defensa del cultivo. Asimismo, de la progresiva desarticulación del gremio cocalero mediante intervenciones policiales de sus principales líderes, bajo el sustento de tener vínculos tanto con el terrorismo como con el mercado ilegal de la cocaína. Como consecuencia, siguiendo las ideas del autor, si bien el Monzón daba cuenta de un campo de poder construido en torno a la economía ilícita de la hoja de coca, la localidad transita ahora hacia un nuevo escenario en el cual el Estado asume un mayor protagonismo en la determinación del orden local. No resulta extraño entonces que, en medio de este proceso de transición, el Estado tenga capacidad de implementar acciones de erradicación de cultivos, en un contexto en el que eran impensables estas intervenciones décadas atrás.

En una línea complementaria, autores como Casas y Ramírez (en esta edición) tratan también la transición del Monzón desde la reconfiguración de las relaciones de poder y el capital político de los actores locales. La constitución de este valle como bastión del movimiento cocalero implicaba en términos simbólicos (aunque también prácticos) una muestra de las debilidades del Estado peruano para implementar la política de drogas. Si bien la lectura oficial de la intervención en el valle resalta factores burocráticos en la consecución de los logros (mayor logística, mayor voluntad política, etcétera), los autores señalan que estos no se pueden mirar sin considerar la situación de dos actores: Sendero Luminoso y el movimiento cocalero. Así, la desarticulación de la organización terrorista implicó la pérdida de una fuerza beligerante involucrada en la defensa de la hoja de coca. Esta desarticulación estuvo acompañada por la pérdida de legitimidad de los dirigentes del gremio cocalero frente a los campesinos. Si a eso se suma como un tercer factor el refuerzo de las intervenciones del Desarrollo Alternativo mediante la incorporación de operadores privados (lo que en cierta medida permitió neutralizar la desconfianza que genera el Estado por sí mismo), se entiende de mejor manera cómo es que se lograron los objetivos de política del control de cultivos. Se trata de un nuevo escenario en el que el Estado tiene mayor margen de autonomía, dado el resquebrajamiento de las resistencias.

Estudios como el de Manrique (en esta edición) plantean una lectura semejante, pero desde la expansión de la presencia del Estado en los territorios donde se desarrolla el cultivo de la hoja de coca. Para ello, propone mirar el denominado «Modelo San Martín» (coloquialmente llamado el «milagro» San Martín), que responde a la sostenida reducción de los cultivos de hoja de coca en el departamento amazónico con el mismo nombre, así como a la 
expansión de las actividades económicas lícitas. Lo que sugiere el autor es que resulta necesario comprender esta intervención desde la construcción de capacidades estatales, centrada en la confluencia de una multiplicidad de actores por, cuando menos, tres décadas de trabajo sostenido. Así, el «modelo» implica una lógica de acumulación de la presencia estatal favorecida por la actuación de las agencias de cooperación internacional, de los gobiernos locales y regionales, el accionar policial (en particular contrasubversivo), que se consolida con lo que los estudios antes señalados observan en la fragmentación de las resistencias locales. Una mirada complementaria de este estudio, y los antes mencionados, da cuenta de una reestructuración del equilibrio de poder a favor del Estado. Asimismo, sugiere que no es posible mirar los avances en localidades como el Monzón, sin mirar previamente el fortalecimiento del Estado en espacios cercanos como San Martín, que forma también parte del eje del Alto Huallaga.

Busnel (en esta edición) plantea una entrada distinta para comprender cómo se construyen los actores locales que defienden el cultivo, a partir de la idea de enraizamiento social. Para esto, propone una comparación entre el movimiento cocalero en el valle de La Convención (ubicado en el Cusco) en el Perú y el Chapare en Bolivia. Desde esta mirada se propone entender cómo factores económicos productivos inciden en la construcción del actor sociopolítico cocalero y su resistencia frente a las medidas de control. Así, aun cuando La Convención ha sido uno de los principales escenarios de las pugnas del movimiento por la reivindicación cultural de la hoja de coca, es la dinámica del mercado la que culmina fragmentando las posibilidades de acción colectiva del movimiento. De hecho, La Convención aparece como el único escenario en el Perú en el que la agenda prioriza el precio de compra de la hoja de coca por parte de la agencia estatal responsable (la Empresa Nacional de la Coca - Enaco) y no otras medidas de control como la erradicación (que no existe) o el Desarrollo Alternativo. Esta pugna por el precio de la hoja de coca permitió construir una plataforma local de resistencia. Sin embargo, lo que afectó la capacidad de acción colectiva del movimiento cocalero en la localidad es la emergencia de nuevas actividades económicas. El uso de canon gasífero para obras de infraestructura, absorbió la mano de obra antes destinada al cultivo, ofreciendo mejores pagos y más estabilidad. Así es como el cultivo de hoja de coca va perdiendo espacio en la economía local y soporte sociopolítico para la acción colectiva. En contraste, en el Chapare la resistencia, además de estar orientada al desarrollo alternativo, sigue siendo soportada en el que el cultivo de hoja de coca se mantiene como principal actividad económica de la localidad. 
Aproximaciones como las que propone Stöckli (en esta edición) plantean una mirada a la implementación cotidiana de las medidas de control de cultivos. Desde la experiencia del desarrollo alternativo en la cuenca del Aguaytía (ubicada en el departamento de Ucayali en la Amazonía peruana), el estudio da cuenta de las estrategias que deben implementar los operadores de los proyectos de desarrollo alternativo, a fin de lograr los objetivos de política propuestos. Cabe indicar que, como señala la autora, se trata de un escenario en el cual existen precedentes negativos sobre esta medida de control de cultivos (que en algún momento implicaron el retiro de oficinas de las agencias del Estado responsables de la materia). En un nuevo contexto, los operadores se ven en la necesidad de aplicar estrategias informales, a pesar de que esta intervención se encuentra protocolizada con bastante detalle. Estas estrategias pueden significar el acercamiento individual informal a las unidades familiares o los jefes de familia de una comunidad, a fin de generar compromisos individuales, sin necesariamente utilizar como mecanismo el diálogo formal inicial con quien lidera la comunidad. También significan la fragmentación de las responsabilidades ante efectos nocivos de otras medidas de control. Por ejemplo, se da cuenta de una práctica que implica diferenciar los proyectos del desarrollo alternativo de las acciones de erradicación, que corresponden a agencias estatales diferentes. Asimismo, estas estrategias también pueden significar flexibilizar los controles sobre el propio cultivo. De esta manera, los operadores se concentran en que el éxito de su programa (y el cumplimiento de sus metas) sea suficiente incentivo para que el agricultor deje el cultivo, sin la necesidad de reportarlo ante las autoridades competentes. Así, este escenario describe un Estado que, desde sus operadores locales, plantea estrategias cotidianas informales que le permiten cumplir con una política de Estado.

Otras miradas, como la de Vizcarra (en esta edición), dan cuenta de las prácticas de legitimación de la hoja de coca a nivel local. A partir del estudio de la representación de esta planta en las obras públicas, señala que no solo se trata de una inscripción de las mismas en el espacio público de uso cotidiano. El discurso que subyace a la construcción de parques y plazas en los que se representa a la hoja de coca, o de actividades feriales con la misma finalidad, implica también una respuesta crítica a la política de drogas, específicamente al control de cultivos. Desde esta mirada, ello significa una proyección del dominio territorial de estos intereses en aquellas localidades donde el cultivo tiene presencia. Ello implica una muestra de la cotidianeidad de las resistencias en localidades en las que el Estado tiene menos alcance y posibilidades de expansión. 
Finalmente, otros estudios plantean estudiar la dinámica de las resistencias locales ante medidas como la erradicación en las principales cuencas cocaleras del Perú. Estos permiten observar, por un lado, cómo el éxito de las resistencias ante el control de cultivos se asocia a la fragmentación de la postura dentro del propio Estado al momento de relacionarse con los actores locales (Zevallos ,2015). En estos casos, se da cuenta de cómo la cercanía de ciertas agencias estatales resistentes a la implementación de medidas de erradicación en valles como el de los ríos Apurímac, Ene y Mantaro (conocido como VRAEM) derivaron en la suspensión de estas actividades, a pesar de los mandatos de política inicialmente previstos. Por otro lado, respecto a la misma medida de control pero en el valle del Monzón, permiten observar cómo las mismas relaciones dentro del propio Estado — esta vez con una postura unificada - fueron centrales para la puesta en marcha de las acciones de erradicación (Zevallos, 2016a). Una lectura complementaria de ambos estudios permite comprender el impacto que tiene el papel de la postura de las agencias estatales en la relación con los actores locales, al momento de implementar medidas de control de cultivos.

En resumen, la literatura expuesta propone, cuando menos, cuatro elementos para discutir las dinámicas locales en torno al control del cultivo de hoja de coca. En primer lugar, el progresivo debilitamiento de las resistencias locales, derivadas de la pérdida de capital sociopolítico (Busnel en esta edición), como de la deslegitimación de las organizaciones que defienden el cultivo (Heuser en esta edición; Casas y Ramírez en esta edición). En segundo lugar, la progresiva expansión de la presencia estatal en las zonas cocaleras, que alimenta la posibilidad de tener mayor autonomía frente a posiciones de defensa débiles (Casas y Ramírez en esta edición; Manrique en esta edición). En tercer lugar, un Estado que, a pesar de contar con mayor fuerza a nivel local, implementa en el cotidiano estrategias informales y flexibles para lograr sus objetivos de política (Stockli en esta edición). Finalmente, escenarios en los que las resistencias locales tienen relativo éxito, sostenidos a partir de la fragmentación de la posición del propio Estado sobre la implementación de medidas de control (Zevallos, 2015, 2016a).

\section{CONSIDERACIONES PARA EL ESTUdio DE LAS DINÁMICAS LOCALES EN TORNO AL CONTROL DEL CULTIVO}

Primera consideración. Como política pública, el control de mercados ilegales como el de la cocaína está condicionado a las relaciones entre el Estado y los actores involucrados en estos fenómenos. De acuerdo al poder que acumulen 
estos actores, estas relaciones pueden representar una limitación efectiva para la autonomía del Estado y, por ende, limitar la efectividad de las medidas de control. Para comprender estas relaciones, es importante conocer qué sentidos subyacen a la construcción de sus agendas de resistencia ante las medidas de política. Un escenario para ello es el control de cultivos de hoja de coca.

Segunda consideración. Actualmente, el cultivo de hoja de coca está fuertemente asociado a la cadena de suministro de la cocaína, pues es esta la que demanda la mayor parte del volumen de producción de este producto vegetal. Eso implica entender el sentido material que subyace a esta actividad agrícola actualmente, que trasciende — mas no resta valor-a la comprensión histórica de la hoja de coca. En ese escenario, las evidencias permiten sostener que no es necesariamente la rentabilidad lo que anima esta actividad agrícola (puesto que el dinero que moviliza es residual si se contrasta con lo generado por otros eslabones de la cadena de suministro de la cocaína). En todo caso, lo que parece animar el cultivo es el sostenido acceso a un mercado global relativamente estable en el tiempo.

Tercera consideración. La configuración de la cadena de suministro de la cocaína da cuenta de la focalización de los cultivos de hoja de coca en la región andina, sostenida en las ventajas competitivas que estos territorios ofrecen para su desarrollo. Es en esta región en la que se han concentrado las plataformas de defensa del cultivo de hoja de coca, cuyas agendas han integrado de manera particular a los beneficios materiales derivados de esta actividad agrícola. En Bolivia, Colombia y Perú esta construcción plantea como elementos comunes que (1) derivan de procesos de colonización de la franja amazónica en los que (2) los Estados no han podido ofrecer alternativas económicas lícitas viables y que (3) han encontrado en una hoja de coca articulada a la cadena de suministro de la cocaína una posibilidad de generación de ingresos. Asimismo, la agenda de las plataformas de defensa ha integrado (4) resistencias a las medidas de control de cultivos, en particular en lo referido a (5) la militarización de las localidades donde esta actividad tiene presencia.

Cuarta consideración. En el caso peruano, la evolución de la defensa del cultivo ha llevado a que, actualmente, el movimiento cocalero tenga poca visibilidad a escala nacional. Sin embargo, las resistencias se mantienen a nivel local en las principales zonas cocaleras. Ello ha implicado algunas particularidades en el desarrollo de las relaciones en torno al control del cultivo. Por un lado, una mayor presencia del Estado frente a las debilidades de los actores locales. Por otro lado, la subsistencia de racionalidades que defienden el cultivo por los beneficios que genera, a pesar de que se reconozca los problemas que implica. 
Asimismo, un Estado que flexibiliza sus posiciones frente a los actores locales, a fin de lograr cumplir con sus objetivos de política.

Quinta consideración. En síntesis, el panorama expuesto propone comprender el sentido que cobra el aspecto material que prima en el cultivo de la hoja de coca al momento de estudiar las relaciones construidas en torno a la defensa de esta actividad agrícola. Si bien ello no implica desconocer los aspectos culturales, sí significa repensar la política de drogas a partir de estas categorías. En ese sentido, pensar las relaciones entre el Estado y los actores locales existentes en torno al cultivo de hoja de coca, requiere no resumir la comprensión de las mismas a las estrategias de acción colectiva. Demanda una mirada integral al papel del cultivo en una cadena extensa y compleja, cuya racionalidad económica trasciende largamente a su carácter ilícito. Ello significa un reto no solo para aproximaciones académicas al fenómeno, sino para el diálogo sobre una política de drogas que comprenda los sentidos que subyacen a estos problemas públicos.

\section{REFERENCIAS}

Allen, C. M. (2005). An Industrial Geography of Cocaine (1 edition). Nueva York: Routledge.

Cabieses, F. (1996). La hoja de coca y sus encrucijadas. Debate Agrario, (25), 149-164. http://www. cepes.org.pe/debate/debate25/08_Articulo.pdf

Cabieses, H. (2005). Coca compleja, drogas y cocaleros en los Andes. En H. Cabieses, B. Cáceres, R. Rumrill y R. Soberón, Hablan los diablos: Amazonía, coca y narcotráfico en el Perú: escritos urgentes (pp. 15-103). Lima, Perú: Abya Yala.

Castillo, M. (2004). Movimiento cocalero en Bolivia. Violencia, discurso y hegemonía. Gaceta de Antropología, (20). http://digibug.ugr.es/handle/10481/7286

CVR-Comisión de la Verdad y la Reconciliación (2003). Tomo V. Capítulo 2. Informe Final. Lima: CVR.

Cortez, R. (1993). Coca y cocaleros en Bolivia. En H. Tovar Pinzón (ed.), La coca y las economías de exportación en América Latina. Andalucía: Universidad Hispanoamericana. Santa María de la Rábida (pp. 125-162). Recuperado a partir de http://dspace.unia.es/handle/10334/1610

De la Peńa, R. (1972). El uso de la coca entre los incas. Revista española de antropología americana, (7), 277-306.

Del Olmo, R. (1975). La sociología politica de las drogas. Caracas: Universidad Centro de Venezuela.

Díaz, A. M. y Sanchez, F. (2004). Geografía de los cultivos ilícitos y conflicto armado en Colombia. Bogotá, Colombia: Centro de Estudios de Desarrollo Económico, Universidad de los Andes. https://core.ac.uk/download/pdf/6517082.pdf

García, E. O. (2008). Coca y minería en el Alto Perú durante el periodo colonial. De Re Metalica, (10), 99-107.

Garzón, E. (ed.). (1993). Significado histórico de la coca en el mundo andino. En H. Tovar Pinzón (ed.), La coca y las economías de exportación en América Latina (pp. 193-202). Huelva, España: Universidad Hispanoamericana Santa María de la Rábida. 
Giraudy, A. (2012). Conceptualizing State Strength: Moving Beyond Strong and Weak States. Revista de Ciencia Politica, 32(3), 599-611. http://www.redalyc.org/articulo.oa?id=32425402005. Fecha de consulta: 31 de mayo de 2017.

Gootenberg, P. (2003). Entre la coca y la cocaina. Un siglo o más de las paradojas de la droga entre Estados Unidos y el Perú, 1860-1980. Lima: Instituto de Estudios Peruanos.

Gootenberg, P. (2006). Cocaine in chains: The rise and demise of a global commodity, 1860-1950. En S. Topik, C. Marichal y Z. Frank, From silver to cocaine, Latin American commodity chains and the building of the World Economy, 1500-2000 (pp. 321-351). Durham y Londres: Duke University Press.

Hinostroza, L. (1985). Breve informe sobre la coca. Anthropologica, 3(3), 153-170. https://dialnet. unirioja.es/servlet/articulo?codigo $=5042017$

INEI-Instituto Nacional de Estadística e Informática (2015). Análisis de los resultados de la Encuesta de Hogares sobre demanda de la Hoja de Coca 2013. Lima: Instituto Nacional de Estadística e Informática. Comisión Nacional para el Desarrollo y Vida sin Drogas.

Kennedy, M., Reuter, P. y Riley, K. J. (1993). A simple economic model of cocaine production. Mathematical and Computer Modelling, 17(2), 19-36. https://doi.org/10.1016/ 0895-7177(93)90237-S

Kenney, M. (2007). From Pablo to Osama: Trafficking and Terrorist Networks, Government Bureaucracies, and Competitive Adaptation. College Park, PA: Penn State Press.

LINCS in Supply Chain Management Consortium (mayo de 2016). Warehousing Operations Certification Track. www.LINCSeducation.org

Lloyd, J. U. y Lloyd, J. T. (1913). Coca "The divine plant of the incas". Journal of the American Pharmaceutical Association 2(10), 1242-1244. https://doi.org/10.1002/jps.3080021005

López, N. (2015). Modelo de estimación de evitabilidad de la cocaína en el mercado mundial: el caso peruano. En J. Mujica y N. Zevallos (eds.), Herramientas y métodos de análisis sobre el tráfico de cocaina (pp. 54-75). Lima: Comisión Nacional para el Desarrollo y Vida sin Drogas, Escuela de Gobierno y Políticas Públicas de la Pontificia Universidad Católica del Perú, Laboratorio de Criminología PUCP.

Mella, R. (2012). La cocaína del VRAE. IDL-Reporteros. https://idlreporteros.pe/la-cocaina-delvrae/. Fecha de consulta: 2 de junio de 2017.

Morales, E. (2002). Las hojas de coca y el cocaísmo en la historia peruana. Lima: Bibliotécnic Consultores. http://biblioteca2.pe.tripod.com/documentos/documento28.pdf

ONU-Organización delas NacionesUnidas. (1961). ConvenciónÚnicade 1961 sobreEstupefacientes. https://www.incb.org/documents/Narcotic-Drugs/1961-Convention/convention_ 1961_es.pdf

ONU-Organización de las Naciones Unidas (1988). Convención de las Naciones Unidas contra el Tráfico Ilícito de Estupefacientes y Sustancias Sicotrópicas de 1988. https://www.unodc.org/ pdf/convention_1988_es.pdf

Pinto, M. T. (2008). De cómo lograr la trascendencia política desde abajo: las movilizaciones cocaleras en Bolivia (1987-2001). Análisis Politico, 21(64), 40-56.

Ramírez, M. C. (2011). Between the Guerrillas and the State: The Cocalero Movement, Citizenship, and Identity in the Colombian Amazon. Durham, NC: Duke University Press.

Silva, R. (2010). Organizaçao política e cultivos ilícitos de coca na Bolivia. Uma abordagem etnográfica. Revista Brasileira de Ciências Sociais, 25(73), 27-40. https://doi.org/10.1590/ S0102-69092010000200002

Thoumi, F. E. (2003). Illegal Drugs, Economy, and Society in the Andes. Washington DC.: Woodrow Wilson Center Press with Johns Hopkins University Pres. 
UNODC-United Nations Office on Drugs and Crime (2016). World Drug Report 2016. Nueva York: Naciones Unidas.

UNODC-United Nations Office on Drugs and Crime (2014). Perú: Monitoreo de coca 2013. Lima: UNODC, Devida.

Uribe, G. y Ferro, J. G. (2014). Las marchas de los cocaleros del departamento de Caquetá, Colombia: contradicciones políticas y obstáculos a la emancipación social. Cuadernos de Desarrollo Rural (Colombia), (49).http://repositoriodigital.academica.mx/jspui/handle/987654321/199184

Van Dun, M. (2009). Cocaleros: Violence, Drugs and Social Mobilization in the Post-Conflict Upper Huallaga Valley, Peru. Tesis de Doctorado. Utrecht University Repository. https://dspace.library. uu.nl/handle/1874/33733

Von Lampe, K. (2016). Organized Crime: Analyzing Illegal Activities, Criminal Structures, and Extralegal Governance. Los Angeles, CA: SAGE Publications, Inc.

Zevallos, N. (2014). Hoja de coca y la Estrategia Nacional de Lucha contra las Drogas 20072011: el problema público en el control de cultivos. Revista de Ciencia Politica y Gobierno, 1(1), $97-114$.

Zevallos, N. (2015). Hacia un modelo analítico de las capacidades estatales en espacios subnacionales: una lectura desde resistencia a la erradicación de hoja de coca en una cuenca cocalera del Perú. Presentado en XXXIII International Congress of the Latin American Studies Association $L A S A$, Puerto Rico.

Zevallos, N. (2016a). Capacidades estatales y resistencias a la erradicación de cultivos: una mirada al Monzón. RITA-Revue Interdisciplinaire de Travaux sur les Amériques, (9). Recuperado de http:// www.revue-rita.com/notesderecherche9/capacidades-estatales-y-resistencias-a-laerradicacionde-cultivos-una-mirada-al-monzon.html

Zevallos, N. (2016b). Control y defensa del cultivo de hoja de coca en el Perú. Lima: Laboratorio de Criminología. Escuela de Gobierno y Políticas Públicas de la Pontificia Universidad Católica del Perú. Recuperado de http://www.revue-rita.com/notesderecherche9/capacidades-estatalesy-resistencias-a-la-erradicacion-de-cultivos-una-mirada-al-monzon.html 